\title{
Sociedades del conocimiento como motor generador de tecnología e innovación agrícola en el Ecuador
}

\section{Knowledge societies as generator of agricultural technology and innovation in Ecuador}

\author{
Carmen Mayorga Villamar ${ }^{1}$ y Rogelio Suarez Mella ${ }^{2}$ \\ ${ }^{1}$ Universidad Autónoma de Los Andes extensión Babahoyo, Babahoyo: Av. Jorge Villegas y tercera \\ transversal \\ 2 Universidad Tecnológica Equinoccial, Quito: Campus Matriz- Quito.Bourgeois N34-102 y Rumipamba
}

DOI: https://doi.org/10.33017/RevECIPeru2015.0016/

\section{Resumen}

El presente estudio, tiene como propósito, el análisis del aporte que producen las sociedades del conocimiento como motor generador de tecnologías e innovaciones en el sector agrícola en la República del Ecuador. Este aporte origina evoluciones en red, en el marco agrícola empresarial e individual, a través del diseño de nuevos procesos productivos y de gestión en la calidad de sus procesos, así como el rediseño de los procesos ya utilizados, que se involucran de carácter social y económico en el contexto de la agricultura ecuatoriana. Basándose en proposiciones, que las sociedades de conocimiento de alto impacto científico agrícola, contribuyen en la mejora del sector agrícola a través de la creación de nuevas tecnologías e innovaciones con características propias del sistema agrario ecuatoriano, dando el despliegue a un marco de crecimiento económico social y cultural del país ecuatoriano.

Hay que determinar el término de "sociedades del conocimiento", en uso de esta investigación, considerándolo como factor del cambio social en la expansión de la educación, y de las competencias específicas de los empleados, que se proyecta con una visión de esfuerzos educativos y formativos.

Anteriormente en el Ecuador, quienes invertían en sociedades de conocimiento como fuente de investigación científica, para el proceso agrícola, eran las empresas privadas, mientras que el sector público realizaba mejoras para el sector agrícola, pero de bajo impacto, debido al alto gasto que genera los programas de desarrollo agrícola para un país, con eficiencia, control y seguimiento de los mismos. Y de los cuales pocos eran los beneficiarios, ya que los programas llegan a los grandes y medianos productores que son los propietarios de la mayor cantidad de tierra productiva en el país, dejando vulnerable a los pequeños agricultores. Esto ha provocado que durante estos últimos años, los resultados obtenidos como contribución del desarrollo rural no hayan sido suficientes, evidenciando alto índice de pobreza y un decreciente desarrollo endógenos en los sectores agrícolas, ya que los efectos generados no han sido útiles para el campesino, productores empresariales, procesadores, comercializadores y exportadores agrícolas.

Los métodos y técnicas empleadas en el presente trabajo científico, son de nivel teórico del conocimiento, tales como : histórico-lógico, inductivo-deductivo, análisis-síntesis para realizar un análisis de las sociedades del conocimiento generadoras de tecnologías e innovación en el sector agrícola ecuatoriano; entre los métodos del nivel empírico del conocimiento: la observación científica para estudiar las características fundamentales del sector agrícola y la contribución de las sociedades de conocimiento. Las técnicas a utilizar son técnicas documentales informativas y argumentativa de campo.

Se puede concluir que esta investigación servirá para que las sociedades de conocimiento a través de la creación de tecnologías e innovación agrícola eleven el grado de desarrollo en las producciones, sino que contribuyan en el desarrollo industrial, para que Ecuador se introduzca en la competencia del mercado interno y externo, apuntalándose a un creciente desarrollo económico y social. 
Descriptores: sociedades del conocimiento, tecnología, innovación, l+D

\section{Abstract}

The present study aims to analyze the contribution that produces knowledge societies as generator of technologies and innovations in the agricultural sector of the Republic of Ecuador. This contribution originates network evolutions in corporate and individual agricultural framework, through the design of new production processes and quality management processes and redesigning processes already used, which involve social and economic aspects in the context of ecuadorian agriculture. Based on propositions that knowledge societies of high scientific agricultural impact, contribute to improve the agricultural sector through the creation of new technologies and innovations directed to the ecuadorian agricultural system characteristics allowing a social, economic and growth in Ecuador.

We must determine the term of "knowledge societies", in used in this research, considering it as a factor of social change in the expansion of education, and specific skills of employees, projecting a vision of educational efforts and training.

Earlier in Ecuador, institutions that invested in knowledge societies as a source of scientific research to the agricultural process were private companies, while the public sector performing agricultural improvement, but low impact, due to the high cost that generates agricultural development programs for a country with efficiency, control and monitor. The beneficiaries of those programs reach the large and medium producers who are the owners of the most productive land in the country, leaving you vulnerable to small farmers. This has meant that in recent years, the results as a contribution to rural development have not been sufficient, showing high levels of poverty and decreasing endogenous development in the agricultural sectors, because the generated effects have not been useful for the farmer, corporate producers, processors, traders and agricultural exporters.

The methods and techniques used in this scientific work are of theoretical knowledge, such as historical and logical, inductive-deductive, analysis-synthesis for analysis of knowledge societies generating technologies and innovation in the agricultural sector Ecuador, between the empirical methods of knowledge: scientific observation to study the fundamental characteristics of the agricultural sector and the contribution of knowledge societies. The techniques used are informative and argumentative documentary techniques of field. We conclude that this research will serve to knowledge societies through the creation of agricultural innovation technologies and raise the level of development in the productions, but contribute to industrial development, that Ecuador is introduced in market competition internal and external bracing a growing economic and social development.

\section{Keywords: knowledge societies, technology, innovation, $R \& D$}

\section{Introducción}

En Ecuador las innovaciones tecnológicas agrícolas han sido de primera generación, lo que ha generado el desarrollo productivo de las tierras, aprovechando cada metro cuadrado en cultivos, siendo estas innovaciones el riego, arado, preparación de la tierra, incorporación de fertilizantes de máximo rendimiento, entre otros.

En el 2010 el Ecuador tuvo un total de 7’303.674 hectáreas de labor agrícola, siendo el $19,5 \%$ de cultivos permanentes y el $13,5 \%$ de cultivos transitorios, dentro de los cultivos permanentes el $40,44 \%$ son caña de azúcar, banano y palma africana y dentro del cultivo transitorio el $80 \%$ corresponde al arroz, maíz y papa [1].

Los cultivos permanentes y transitorios llegan al consumidor a granel, porque la producción agrícola del Ecuador carece de tecnologías e innovación para transformar la materia prima en productos procesados y hacer de las empresas agrícolas (haciendas, fincas, hectáreas de mono y pluricultivos) industrias agrícolas procesadoras de alimento.

Como antecedentes de la investigación se encuentra que el Ecuador dentro de los países de América Latina ocupa el puesto $14^{\circ}$ sobre 18 puestos con un índice de economía de conocimiento del 3,46\%, siendo Chile el país con mayor aporte del 6,92\% [2]. Puesto que las universidades del país no se encuentran en las 500 mejores universidades del mundo, según Academic Ranking of World Universities en el 2013, existe una reducida sociedad de conocimiento generadora de tecnología e innovación agrícola, por falta de impulso científico hacia los estudiantes, para que realicen investigación en el área agrícola.

Las tecnologías e innovación agrícola que existen en el país, son fuentes de materias primas de países desarrollados, que generan tecnología e innovación 
de alto impacto competitivo, para extraer o importar productos agrícolas y poder transformarlos, dándole un valor agregado.

Esta investigación justifica el estudio, debido a que es necesario conocer el estado de arte de las sociedades de conocimiento cuando se empoderan de los recursos que poseen y que es necesario investigarlo para poder transformarlo y evolucionarlo aprovechando las ventajas competitivas que poseen y así contribuir en el desarrollo económico y social del país caso de estudio.

El objetivo general de la investigación, es determinar el estado actual de la sociedad del conocimiento y su aporte a la agricultura con nuevas tecnologías e innovaciones, lo que llevará a establecer como objetivos específicos, la identificación de las características y particularidades de la sociedad del conocimiento en el Ecuador, analizar los procesos tecnológicos e innovaciones agrícolas existentes en el país y valorar el aporte de las sociedades de conocimiento al desarrollo agrícola del país.

Durante el gobierno de la Revolución Ciudadana en el ecuador, el sector agrícola ha tenido cambios radicales en cuanto a producción y rendimiento agrícola se refiere, ya que el gobierno ha desarrollado programas agrícolas y asesorías de apoyo agrario para solventar las contingencias presentadas en el sector por problemas naturales, que han ocasionado incertidumbres entre los agrícola. Este sector de la economía, se ve afectado en ocasiones por las escasas o incrementadas lluvias, que provocan perdidas en los cultivos.

Las universidades en Ecuador están pasando por un proceso de acreditación y evaluación, para medir la calidad de la enseñanza que presentan. Dentro de los productos y servicios que estas brindan, se encuentra la investigación científica creadora de conocimiento científico y tecnológico. Con el fin de llegar a desarrollar sociedades de conocimiento aptas para cubrir las necesidades que se demanden en este mundo cambiante. La inversión que destina el estado ecuatoriano a la educación superior, corresponde al 1,8 \% del PIB, hoy uno de los más altos del mundo.

El ente regulador de la educación superior en conjunto con otras entidades rectoras del progreso y desarrollo de los habitantes, han diseñado nuevas bases legales, para implementarlas en la educación y así estas puedan alcanzar un alto nivel científico que ayuden al crecimiento de la nación.

\section{Particularidades de la sociedad del conocimiento en Ecuador.}

La educación superior juega un rol importante en la sociedad del conocimiento, ya que a través de la formación de profesionales para que se incorporen a la producción y los servicios, necesitan lograr el enriquecimiento innovador y emprendedor de los mismos, por medio del proceso investigativo de la carrera a seguir. La sociedad del conocimiento es un punto débil focal en el Ecuador, no solo por parte de las instituciones educativas, sino por el compromiso de investigación de las empresas privadas y entidades públicas.

El rubro en $\mathrm{I}+\mathrm{D}+\mathrm{i}$ visto desde el sistema de innovación de triple hélices: Universidad, empresa y gobierno, se encuentra en bajo porcentaje de aportación, porque no se aplican las políticas públicas de promoción investigativa a estas 3 entidades. Estas entidades utilizan cerebros importados para generar investigación, o adquieren tecnología proveniente de los países desarrollados, para dar valor agregado a los productos o servicios que estas brindan. Según el Plan Nacional del Buen Vivir en el año 2011 en Ecuador solo existían tres revistas indexadas en Bases de Datos Internacionales, cuestión esta que revele el bajo índice de generación de artículos científicos que generaban los actores de la académica y de la ciencia. En el caso de las universidades ecuatorianas en el 2012 existían 431 profesionales académicos con Doctorados o Phd, obtenidos sus títulos en un $6,7 \%$ de universidades nacionales y el $93,3 \%$ de universidades extranjeras, es decir que las universidades contaban con el $1,5 \%$ de profesores de quinto nivel académico.

El verdadero desarrollo de los pueblos es realizar investigación científica de la realidad de un país, para así poder generar conocimientos autóctonos propios y característicos de los problemas fehacientes que presenta la sociedad, no solo con importar conocimientos que no pueden descifrar la realidad nacional, para perfeccionar las técnicas y difundirlas a la sociedad de conocimiento para que sean reproducidas por los ciudadanos.

Dentro del nuevo reglamento de educación superior, los docentes titulares deben obtener el título de Phd hasta el 2017, generándose una gran demanda de profesionales que desean realizar el estudio de quinto nivel. Es por ello que el gobierno nacional ha concedido becas a docentes, profesionales $y$ estudiantes para que obtengan sus títulos en universidades nacionales o extranjeras, con la reciprocidad de que vuelvan al país a contribuir con los conocimientos adquiridos.

El sistema educativo en general, es poco adaptable a nuevas tecnologías, es necesario rediseñar un sistema flexible al cambio, ya que las tecnologías e innovaciones tiene cambio constantemente y es necesario que el sistema educativo se incorpore a ellos, obteniendo resultados a un mediano y largo 
plazo en conjunto con profesionales investigadores que aporten con el desarrollo de capacidades y competencias para una excelencia académica.

El Ecuador ha determinado un nuevo marco legal en el cual se desenvuelven las Universidades, la Constitución Política de la República del Ecuador (2008), el Plan Nacional del Buen Vivir (PNBV 2009), la Ley Orgánica de Educación Superior (LOES - 2010), como política pública social examina fortalecer la calidad de las universidades. El nuevo sistema de investigación científica a través de los entes rectores como el Sistema Nacional de Ciencia Tecnología Innovación y Saberes Ancestrales (SNCTIS), coordinado por la Secretaría Nacional de Educación Superior, Ciencia, Tecnología e Innovación (SENESCYT), Consejo de Evaluación, Acreditación y Aseguramiento de la Calidad de la Educación Superior (CEAACES - 2010), el Consejo de Educación Superior (CES - 2010) y la Secretaria Nacional de Planificación y Desarrollo (SENPLADES - 2010), integran a las universidades a obtener resultados científicos para el desarrollo económico, político y social del país, mediante la generación y aplicación de tecnologías innovadoras en procesos productivos que se vuelvan sostenibles a través del tiempo y contribuyan a mejorar la calidad de vida de los ecuatorianos.

Por tanto, el gobierno nacional actual, busca introducir sociedades de conocimiento para lograr articular con otros grupos de investigación, estrategia que asegure la transferencia de conocimientos, tecnologías y resultados de gran impacto sobre las capacidades institucionales en investigación y desarrollo en la formación del talento humano y fortaleciendo las base científico - técnica de las universidades.

Los organismos rectores de investigación científica tendrán que ejecutar proyectos sobre áreasproblemas o prioridades identificadas, de acuerdo a las áreas y líneas de $(I+D)$ definidas por las agendas sectoriales. Para incrementar el conocimiento disciplinario disponible a nivel nacional, dándole solución a los problemas de carácter multidisciplinario con alcance territorial nacional.

\section{Tecnología e innovación en el sector agrícola ecuatoriano.}

Particularmente las investigaciones agrícolas en el Ecuador, se orientan para obtener la mayor productividad para los productos de exportación, los cuales constituyen un factor determinante en la dinámica de la economía ecuatoriana, pero no se ha considerado generar estrategias para el desarrollo tecnológico e innovador destinado a los pequeños productores que son los que proveen de alimentos básicos y de los cuales depende la seguridad alimentaria del país. El Ecuador es un país rico en recursos naturales, no solo aquellos que han sido habitualmente claves para el desarrollo, como los productos agrícolas y mineros, sino aquellos que serán claves en el futuro, como es el agua y la riqueza genética contenida en ecosistemas mega diversos.

La investigación con mayor acento en la experimentación y adecuación de prácticas de bajo costo y de menor riesgo (selección de semilla local, épocas de siembra, densidad de plantas, mejor uso de materia orgánica) ha sido más aceptada por los pequeños agricultores.

La innovación tecnológica está asociada significativamente a capital e insumos, por ello es un marco de innovación muy restrictivo. El manejo integrado de cultivos y escalamiento de bioinsumos son los resultados de estas actividades agrícolas, basados en el conocimiento que por tradición tienen los productores de sus cultivos y su manejo. En ellas se resaltan las iniciativas de los pequeños productores en la construcción y dotación de laboratorios para la multiplicación y uso de insectos y microorganismos biocontroladores, el uso de labranzas de conservación en la preparación de suelos, así como el de variedades nativas o introducidas con alto potencial de tolerancia a plagas y enfermedades que son limitantes en los sistemas de producción. La aplicación de trampas para medir las dinámicas poblacionales de insectos y la utilización de extractos botánicos para el control de plagas y enfermedades. Se destaca también el uso de lombriabonos y bocashi como abonos orgánicos y el de micorrizas y violes como biofertilizantes; el uso de herramientas, materiales y maquinaria (como hornos y molinos) para optimizar los procesos de post cosecha, entre otros. Tecnologías que no solo han permitido un manejo integrado de los cultivos, sino que han generado impactos adicionales a la producción como el mantenimiento y conservación del medioambiente.

En el Ecuador, el número de productores que utiliza innovación tecnológica es 27.651 (maquinaria, herramientas, manejo agroecológico del suelo, biocontroladores, desinfección y tratamiento de semillas, tipo y manejo de podas, manejo integrado de plagas y enfermedades, manejo post cosecha), a nivel nacional. La superficie cultivada con bioinsumos 57.455 hectáreas (biol, te de estiércol, compost, humus, insecticidas botánicos, entre otros), a nivel nacional.

La biotecnología ofrece importantes beneficios a la sociedad mediante el mejoramiento de la calidad de vida. Estos beneficios sobresalen en favor del 
desarrollo económico, salud, agricultura, industrial, medioambiente, y bienestar social. En el país la biotecnología y la nanotecnología es aplicada en un $15 \%$ por empresas agrícolas que ayudan con el incremento de las cosechas, la calidad alimentaria (agricultura, acuicultura y ganadería), la producción farmacéutica, el diagnóstico de patógenos y la biorremediación, entre otros. Sin embargo la agricultura limpia: protección genética y biológica, manejo orgánico, uso racional de pesticidas, conservación de suelo y agua, es frecuente en las actividades agrícolas.

En el sector agrícola durante las últimas décadas, la innovación tecnológica ha tenido mayor apunte con lo que respecta a: semillas de variedades nuevas, mejora de tejidos, equipos y técnicas de cultivo. Acompañados de protocolos de calidad, mejoras de gestión, accesos a nuevos mercados y productos.

Sin embargo el sector agrícola carece de reequipamiento y readecuación de la infraestructura de centros de investigación y generación de nuevas tecnologías. A continuación se enlista varios factores que están limitando el mejoramiento de los cultivos por falta de investigación científica, introducción de innovación tecnológica e inversión:

*Variedades de semillas con características demandadas por el sector agrícola, *Híbridos de semilla de alto rendimiento, "Biotecnología en programas de mejoramiento genético, *Ampliar la base genética de los nuevos cultivos de semilla en función del método de selección recurrente, *Intensificar la Introducción y/o intercambio de germoplasma con Programas Nacionales de países productores y Centros Internacionales de Investigación, *Dosis óptimas económicas de nutrición en el cultivo de semilla, *Mejoras de suelo en diferentes sistemas de producción de semilla, *Métodos de utilización eficiente de los fertilizantes, *Sistema Intensivo del Cultivo de semillas, *Tecnología para manejo de la salinidad en el cultivo de semilla, *Patrones locales para interpretar resultados del análisis de suelo y planta, con fines de fertilización, *Metodología sencilla de muestreo

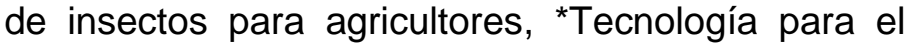

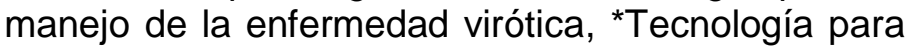
el manejo de enfermedades, en el cultivo del semilla, *Evaluación de la selectividad y eficacia de nuevos herbicidas para el combate de malezas especies gramíneas en condiciones de riego, *Estudios de la capacidad alelopática de diferentes materiales de semilla frente a las principales malezas, *Evaluación del impacto ambiental de las actuales recomendaciones químicas para combate de malezas en semilla de secano y riego, *Láminas y frecuencias de riego, *Compilación y análisis de la información sobre caracterización física de suelos en zonas de riego, *Compilación y análisis de la información de costos de manejo de agua, *Capacitación a técnicos de transferencia de tecnología agrícola, mediante la metodología de Capacitación a Capacitadores, para la aplicación del Manejo Integrado de Cultivo (MIC), *Capacitación a productores líderes con la metodología de Escuelas de Campo (ECA), para difundir y promover la aplicación del Manejo Integrado de Cultivo (MIC), *Desarrollo de metodologías de transferencia de tecnología con base a sistemas participativos, *Negociación y alianzas con Gobiernos locales, Gremios y otras instancias para difundir tecnologías de manera sostenidas, a través de recursos locales, *Producción semilla de calidad y de bajo costo, *Promoción del uso de semilla certificada a través de capacitación, transferencia y divulgación, ${ }^{*}$ Fortalecimiento de la asociaciones con empresas privadas (Gremios y agricultores) para establecer mecanismos institucionales que incrementen el uso de variedades mejoradas y semillas, según parámetros de la legislación vigente.

Con la aplicación y desarrollo de metodologías, estrategias, innovaciones científicas, mediante la investigación científica, mejorará el rendimiento de los cultivos, obteniendo productos de calidad, con la utilización de insumos amigables con el medio ambiento y así salvaguardar la seguridad alimenticia de la población.

\section{Valoración crítica del aporte de las sociedades de conocimiento al desarrollo agrícola del país.}

La Constitución de la República del Ecuador en unas de sus secciones determina que: EI Estado fomentará la ciencia y la tecnología, ....... dirigidas a mejorar la productividad, la competitividad, el manejo sustentable de los recursos naturales y satisfacer las necesidades básicas de la población.........La investigación científica y tecnológica se llevará a cabo en las universidades, escuelas politécnicas, institutos superiores técnicos y tecnológicos y centros de investigación científica, en coordinación con los sectores productivos cuando sea pertinente, y con el organismo público que establezca la ley, la que regulará también el estatuto del investigador científico.

Las sociedades de conocimiento han sido el motor generador de tecnología e innovación en los países desarrollados, iniciadas desde la revolución industrial hasta la actualidad. En el caso de Ecuador las sociedades de conocimiento han aportado al desarrollo agrícola de innovación agrícola pero de manera directa con aplicación de técnicas, y en pocos casos de forma mecánica (creación de 
equipos y maquinarias agrícolas) que faciliten al agricultor en la producción de manera eficiente, eficaz y de calidad.

Los aportes tecnológicos e innovadores han sido aporte de mecanismos creados por el propio agricultor, por la necesidad de mejor sus cultivos de manera empírica o por costumbres ancestrales, adaptándolos a las características propias del cultivo, que han sido utilizados en otros tipo de cultivos, según el clima, suelo, y condiciones de siembra.

El país se ha destacado por la producción y exportación de ciertos productos básicos demandados a nivel mundial como es el cacao y café desde 1880 y el banano (1948), siendo el país el principal exportador a nivel mundial, aportando en el desarrollo económico del país. Sin embargo el aporte de las sociedades de conocimiento como son las universidades y centros de investigación tanto públicos como privados, se han especializado en mejorar la producción del cultivo, procesos, especializar a la mano de obra, dejando a un lado la transformación del producto para darle un valores agregado.

Las provincias del Guayas, Los Ríos, Manabí, se han caracterizado por ser provincias netamente agrícola, y sus economías se basan en la producción agrícola, en estas provincias las sociedades de conocimiento provenientes de las universidades, se especializan en los cultivos básicos existentes, más no en la especialización de cultivar nuevos productos y tecnificarlos.

El ente rector de la educación superior Senescyt, propone a las instituciones de educación preparar a las sociedades de conocimiento en nuevas pertinencias de utilidad con las necesidades propias del país. En el caso específico de la agricultura, a aplicar nuevas tecnologías en los cultivos, mecanizar la producción y realizar variedades de cultivos con la biotecnología en semilla de alta calidad con bajo costo de producción, así como la utilización de productos amigables con el medio ambiente.

Las sociedades de conocimiento en el país están pasando por un proceso de transformación científica, que tiene como objetivo conocer la problemática del país y proponer propuestas para su solución. En el contexto agrícola, las sociedades de conocimiento tienen que ir a la punta de nuevos descubrimientos científicos agrícolas que están siendo estudiados y aplicados en otros países, así como la creación de nuevas tecnologías innovadoras para mejorar la producción agrícola del país y contribuir con el desarrollo del mismo.

\section{Resultados y discusión}

Para que existan sociedades de conocimiento en Ecuador, es necesario que las universidades, centros de investigación 0 institutos realicen investigación científica para generar un conocimiento generador de innovación, tecnología logrando el emprendimiento decisivo para potenciar el desarrollo económico en un país.

\section{Conclusiones}

Las tecnologías e innovaciones son el resultado constante de investigaciones por parte de las sociedades de conocimiento que permiten a los pueblos avanzar, logrando la integración de los factores políticos, económicos y culturales, para que las sociedades de conocimiento sean las portavoces de las nuevas transformaciones en el sistema productivo.

\section{Agradecimientos}

A la familia que es el apoyo constante para alcanzar la meta

\section{Referencias}

[1] Encuesta de Superficie de Producción Agrícola Continua (ESPAC). Superficie de labor agrícola 2010. www.inec.gob.ec. 2011.

[2] J. Brunner. Educación superior en América Latina (2010) 4.

[3] Plan Nacional del Buen Vivir .SENPLADES.Ecuador.2013

E-mail: cmmv11@hotmail.com 